\title{
Phenotypic and genetic characterization of MERS coronaviruses from Africa to understand their zoonotic potential
}

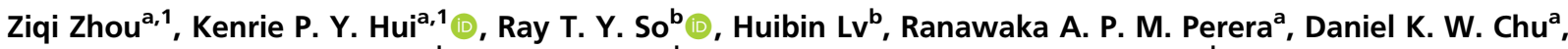

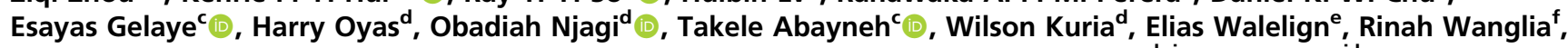 \\ Ihab El Masry' ${ }^{g}$, Sophie Von Dobschuetz ${ }^{\mathrm{g}}$, Wantanee Kalpravidh ${ }^{\mathrm{g}}$, Véronique Chevalier ${ }^{\mathrm{h}, \mathrm{i}}$, Eve Migueli,k, \\ Ouafaa Fassi-Fihri', Amadou Trarore ${ }^{m}$, Weiwen Liang ${ }^{b}$, Yanqun Wang ${ }^{n}$, John M. Nicholls ${ }^{\circ}{ }^{\circ}$, Jincun Zhaon, \\ Michael C. W. Chan ${ }^{a}$, Leo L. M. Poon ${ }^{a, b}$, Chris Ka Pun Mok ${ }^{b, p, 2}{ }_{(0)}$, and Malik Peiris ${ }^{a, b, 2}$
}

\begin{abstract}
aSchool of Public Health, Li Ka Shing Faculty of Medicine, The University of Hong Kong (HKU), Pokfulam, Hong Kong Special Administrative Region, People's Republic of China; 'bKU-Pasteur Research Pole, School of Public Health, Li Ka Shing Faculty of Medicine, The University of Hong Kong, Hong Kong Special Administrative Region, People's Republic of China; ${ }^{C}$ National Veterinary Institute, Debre Zeit, Ethiopia; ${ }^{d}$ Directorate of Veterinary Services, Nairobi, Kenya; ${ }^{\mathrm{e}}$ Food and Agriculture Organization, Emergency Centre for Transboundary Animal Diseases, Addis Ababa, Ethiopia; ${ }^{{ }}$Food and Agriculture Organization, Emergency Centre for Transboundary Animal Diseases, Nairobi, Kenya; ${ }^{9}$ Food and Agriculture Organization of the United Nations, Rome

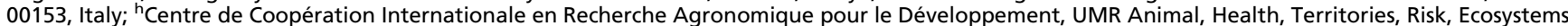
(ASTRE) Animal, Santé, Territoires, Risques et Ecosystèmes, Institut National de la Recherche Agronomique, Université de Montpellier, F-34398 Montpellier, France; 'Epidemiology and Public Health Unit, Institut Pasteur du Cambodge, Phnom Penh, Cambodia; 'ंAnimal, Santé, Territoires, Risques et Ecosystèmes, Centre de Coopération Internationale en Recherche Agronomique pour le Développement, Institut National de la Recherche Agronomique, Université de Montpellier, F-34398 Montpellier, France; ${ }^{k}$ Maladies Infectieuses et Vecteurs: Ecologie Genetique, Evolution et Controle, L'Institut de Recherche pour le Developpment, CNRS, 34090 Montpellier, France; 'Laboratoire de Biologie et Santé Animals, L'Institut de I'Environnement et de Recherches Agricoles du Burkina Faso/Centre National de la Recherche Scientifique et Technologique, 04 BP 8645 Ouagadougou 04, Burkina Faso; ${ }^{m}$ Institut Agronomique et Vétérinaire, Hassan II Université, B.P. 6202 Rabat-Instituts, Rabat, Morocco; ${ }^{n}$ State Key Laboratory of Respiratory Disease, National Clinical Research Center for Respiratory Disease, Guangzhou Institute of Respiratory Health, First Affiliated Hospital of Guangzhou Medical University, Guangzhou, People's Republic of China; ${ }^{\circ}$ Department of Pathology, Li Ka Shing Faculty of Medicine, The University of Hong Kong, Pokfulam, Hong Kong Special Administrative Region, People's Republic of China; and PThe Jockey Club School of Public Health and Primary Care, The Chinese University of Hong Kong, Hong Kong Special Administrative Region, People's Republic of China
\end{abstract}

Contributed by Malik Peiris, May 4, 2021 (sent for review March 4, 2021; reviewed by Kanta Subbarao and John Watson)

Coronaviruses are pathogens of pandemic potential. Middle East respiratory syndrome coronavirus (MERS-CoV) causes a zoonotic respiratory disease of global public health concern, and dromedary camels are the only proven source of zoonotic infection. More than $70 \%$ of MERS-CoV-infected dromedaries are found in East, North, and West Africa, but zoonotic MERS disease is only reported from the Arabian Peninsula. We compared viral replication competence of clade A and B viruses from the Arabian Peninsula with genetically diverse clade $\mathrm{C}$ viruses found in East (Egypt, Kenya, and Ethiopia), North (Morocco), and West (Nigeria and Burkina Faso) Africa. Viruses from Africa had lower replication competence in ex vivo cultures of the human lung and in lungs of experimentally infected human-DPP4 (hDPP4) knockin mice. We used lentivirus pseudotypes expressing MERS-CoV spike from Saudi Arabian clade A prototype strain (EMC) or African clade C1.1 viruses and demonstrated that clade C1.1 spike was associated with reduced virus entry into the respiratory epithelial cell line Calu-3. Isogenic EMC viruses with spike protein from EMC or clade C1.1 generated by reverse genetics showed that the clade C1.1 spike was associated with reduced virus replication competence in Calu- 3 cells in vitro, in ex vivo human bronchus, and in lungs of hDPP4 knockin mice in vivo. These findings may explain why zoonotic MERS disease has not been reported from Africa so far, despite exposure to and infection with MERS-CoV.

MERS-CoV | coronaviruses | Africa | phenotype | characterization

Coro oronaviruses cross species barriers to cause zoonotic and pandemic diseases. While COVID-19 is the most recent example of this $(1,2)$, seasonal human coronaviruses $229 \mathrm{E}$ and OC43 crossed to humans from camelids and bovids in the last few centuries and are now endemic in the human population $(3,4)$. SARS emerged from bats to spread worldwide in $2003(5,6)$. Middle East respiratory syndrome coronavirus (MERS-CoV), first recognized in 2012, is a zoonotic respiratory disease of global public health concern, and dromedary camels are the source of zoonotic infection $(7,8)$. While many zoonotic MERS infections

\section{Significance}

The absence of zoonotic MERS-CoV in Africa in spite of an abundance of MERS-CoV-infected dromedaries has remained an enigma. We demonstrate that geographically and genetically distinct viruses from Africa have low replication competence in the human lung, providing a possible explanation for the absence of severe MERS disease in Africa. The findings suggest that MERS-CoV now entrenched in the Arabian Peninsula has acquired increased pathogenic potential for humans. We demonstrate that the spike protein contributes to this phenotypic difference. If pathogenic clade B viruses from the Arabian Peninsula are introduced into Africa, they are likely to become dominant, as they have in the Arabian Peninsula, and to be associated with adverse health impacts in Africa and increased pandemic threat.

Author contributions: Z.Z., I.E.M., S.V.D., V.C., E.M., C.K.P.M., and M.P. designed research; Z.Z., K.P.Y.H., R.T.Y.S., H.L., R.A.P.M.P., D.K.W.C., E.G., H.O., O.N., T.A., W. Kuria, E.W., R.W., I.E.M., S.V.D., W. Kalpravidh, V.C., E.M., O.F.-F., A.T., W.L., Y.W., J.M.N., J.Z., M.C.W.C., L.L.M.P., and C.K.P.M. performed research; O.F.-F. and A.T. contributed new reagents/analytic tools; Z.Z., K.P.Y.H., H.L., R.A.P.M.P., D.K.W.C., E.G., H.O., O.N., T.A., W. Kuria, E.W., R.W., I.E.M., S.V.D., W. Kalpravidh, V.C., E.M., O.F.-F., A.T., W.L., Y.W., J.M.N., J.Z., M.C.W.C., L.L.M.P., C.K.P.M., and M.P. analyzed data; Z.Z., S.V.D., C.K.P.M., and M.P. wrote the paper; and all authors contributed to revising versions of the paper.

Reviewers: K.S., Peter Doherty Institute; and J.W., Centers for Disease Control and Prevention.

Competing interest statement: Together with other global opinion leaders, M.P. and K.S. coauthored a perspectives article on optimizing the use of the ferret experimental model for research on influenza [J. A. Belser et al. Emerg. Infect. Dis. 24, 965-971 (2018)].

This open access article is distributed under Creative Commons Attribution-NonCommercialNoDerivatives License 4.0 (CC BY-NC-ND).

'Z.Z. and K.P.Y.H. contributed equally to this work.

${ }^{2}$ To whom correspondence may be addressed. Email: kapunmok@cuhk.edu.hk or malik@ hku.hk.

This article contains supporting information online at https://www.pnas.org/lookup/suppl/ doi:10.1073/pnas.2103984118/-/DCSupplemental.

Published June 7, 2021. 
have been sporadic, some have led to large outbreaks of humanto-human transmission involving over 100 individuals $(9,10)$. MERS-CoV was identified by the World Health Organization as an emerging infectious disease of greatest concern for global public health and one for which development of countermeasures is urgently needed (11). The virus is enzootic in dromedary camels in the Arabian Peninsula and the Middle East where zoonotic disease has been reported, but also in East, North, and West Africa, where over $70 \%$ of MERS-CoV-infected dromedaries are found $(12,13)$. So far, however, zoonotic MERS disease has not been detected in Africa and the reason for this is unclear. The prevalence of MERS-CoV infection in dromedaries in Africa is as high as that seen in the Arabian Peninsula $(12,13)$ and the human exposure to infected dromedaries is also substantial (14). Serologic evidence of human MERS-CoV infection in Africa, though detectable, is less common than that observed in the Arabian Peninsula (15-17).

Phylogenetically, three clades A, B, and C, of MERS-CoVs are recognized $(18,19)$. The earliest MERS-CoV virus detected in Saudi Arabia (human/EMC/2012) (EMC) designated as clade A is no longer is detectable in humans or in dromedaries. Clade $\mathrm{B}$ viruses emerged to become dominant in dromedaries in the Arabian Peninsula, spilling over to cause sporadic zoonotic disease, sometimes leading to clusters of transmission between humans. Viruses detected in camels in East (Egypt, Ethiopia, Sudan, Djibouti, and Kenya), North (Morocco), and West (Nigeria and Burkina Faso) Africa all belonged to different sublineages of clade $C(18,19)$. Preliminary studies comparing the replication competence of human (clade A) and camel (clade B) MERS-CoV from Saudi Arabia with a clade $C$ virus from Burkina Faso, West Africa, showed that the West African viruses had lower replication competence in Calu-3 cells, in ex vivo cultures of human bronchus and lung, and in lungs of hDPP4-transduced mice (18). These West African clade C1.1 viruses had deletions in ORF4b that may contribute to the reduced virus replication competence. It was not known whether viruses from other regions of Africa, such as East Africa, where ORF4b deletions are not always found, also share this phenotype of reduced replication in human respiratory cells. Importantly, the highest density of dromedary camels in the world is found in East Africa, which is also the source of export of camels into the Arabian Peninsula. Thus, a more extensive and systematic phenotypic comparison of MERS-CoV from different sublineages and regions of Africa (especially East Africa) with viruses from the Arabian Peninsula is urgently needed. Furthermore, the viral genetic determinants of these phenotypic differences need to be explored.

Here we compare MERS-CoV from West, North, and East Africa with those from the Arabian Peninsula and demonstrate that viruses from Africa had lower replication competence in the human bronchus and lung epithelium and mouse lung. We provide evidence suggesting that the spike protein may be one of the factors contributing to this low-replication phenotype.

\section{Result}

Clade C MERS-CoV from West, North, and East Africa Shows a Lower Replication Phenotype in Ex Vivo Cultures of Human Lung Compared with Clade A and B Viruses from the Arabian Peninsula. The MERSCoVs investigated in this study are listed in SI Appendix, Table $\mathrm{S} 1$ and their phylogenetic relationships are shown in Fig. 1. All virus sequences were derived from virus RNA extracted from the original swab specimen. Some clade $\mathrm{C}$ viruses have diverse deletions in ORF3 and ORF4b (indicated in Fig. 1 and $S I A p$ pendix, Table S2). The virus from Egypt (C270) had no deletions, while the viruses from Ethiopia (CAC9690) and Kenya (CAC10200) characterized in this study had no deletion in ORF4 but had a 12-nucleotide deletion in ORF3. Except for the prototype human virus EMC, all camel viruses used in this study were isolated from swab specimens from dromedary camels in our laboratory in Vero cells using the same methods as previously described (18). The cell culture isolates were genetically sequenced as previously described (20) and any amino acid changes in the culture isolate compared with the virus sequence in the original swab specimen are shown in SI Appendix, Table S3.

We first compared the replication competence of these viruses in ex vivo cultures of human bronchial and lung tissues (Fig. 2 and SI Appendix, Fig. S1). All viruses were tested in lung tissues from each donor and with multiple biological replicates being carried out using different tissue donors. In human bronchus and lung, both human/EMC/2012 (EMC) (clade A) and camel/AlHasa-KFU-HKU13/2013 (AH13) (clade B) viruses from Saudi Arabia exhibited significantly higher replication compared with clade C1.1 viruses isolated from Burkina Faso (camel/Burkina Faso/CIRAD-HKU785/2015) (BF785), clade C2 viruses from Ethiopia (camel/Ethiopia/HKU-CAC9690/2019) (CAC9690) and Kenya (camel/Kenya/HKU-CAC10200/2020) (CAC10200) (Fig. 2), clade C1.1 viruses from Nigeria (camel/Nigeria/NV1657/2016) (Nig1657) and Morocco (camel/Morocco/CIRAD-HKU213/2015) (Mor213), and a clade 1.2 virus isolated in Egypt (camel/Egypt/ NRCE-HKU270/2013) (C270) (SI Appendix, Fig. S1). The immunohistochemical staining of the ex vivo cultures showed that the cell tropism of the different viruses for bronchial and lung tissues were comparable with each other (SI Appendix, Fig. S2 A and $B$ ). The difference in replication kinetics observed in ex vivo cultures of human lung was recapitulated in Calu-3 cells, a cell line of a human respiratory epithelial origin derived from lung adenocarcinoma (SI Appendix, Fig. S3).

\section{Clade C MERS-CoV from West, North, and East Africa Shows a Lower} Replication Phenotype in the Lung of Human-DPP4 Knockin Mice. Previous studies have shown that wild-type MERS-CoV can replicate efficiently in the lung of hDPP4 knockin mice but that these mice do not show evidence of overt disease or weight loss (21). We compared replication kinetics of the clade A, B, and C MERS-CoVs in lungs of hDPP4 knockin mice following intranasal infection. As expected, there were no signs of overt clinical illness in mice infected by different clades of MERS-CoV. However, we found that clade $\mathrm{C} 2$ viruses from Kenya camel/Kenya/ CAC10200/2020 (CAC10200) and Ethiopia camel/Ethiopia/ CAC9690/Ethiopia/2000 (CAC9690) had lower levels of replication than clade A EMC and clade $\mathrm{B}$ AH13 viruses at day 3 postinfection (Fig. $3 A$ and $B$ ).

At day 3 postinfection, the level of virus replication in the lung of the mice infected by EMC (clade A) and AH13 (clade B) from Saudi Arabia were significantly higher (around 100-fold) than those infected by the three African clade C MERS-CoV isolates (Fig. 3A). At day 5 postinfection, only clade C2 CAC10200 virus had lung titers that remained significantly lower than the clade $\mathrm{A}$ and B viruses (Fig. $3 B$ ). Similarly, in a separate experiment, the viruses isolated from clades A (EMC) and B (AH13) from Saudi Arabia showed significantly higher (around 100-fold) levels of replication in mouse lungs when compared with the clade $\mathrm{C} 1.1$ viruses BF785, Nig1657, and Mor213 or a clade C1.2 virus C270 from Egypt (Fig. $3 C$ ). At day 5 postinfection, we did not find a statistically significant difference of virus replication titers among the viruses of clades $\mathrm{A}, \mathrm{B}$, and $\mathrm{C}(P<0.05)$; however, half of the mice (6 of 12) infected by the clade $C$ viruses showed undetectable levels of virus titer in their lungs $(1 / 3 \mathrm{BF} 785,3 / 3$ Nig1657, 1/3 Mor213, and 1/3 C270) while all mice infected with clade $\mathrm{A}$ or $\mathrm{B}$ viruses had virus titers of $>3 \log 10$ tissue culture infectious dose (TCID) $)_{50} / \mathrm{mL}$ (Fig. $3 D$ ). BF785 virus served as an African virus control between the two experiments and provided comparable data in both sets of experiments.

Role of MERS-CoV Spike Protein in Phenotype Differences. Coronavirus spike protein determines the efficiency of virus attachment and entry into the cell and is thus a crucial determinant of 


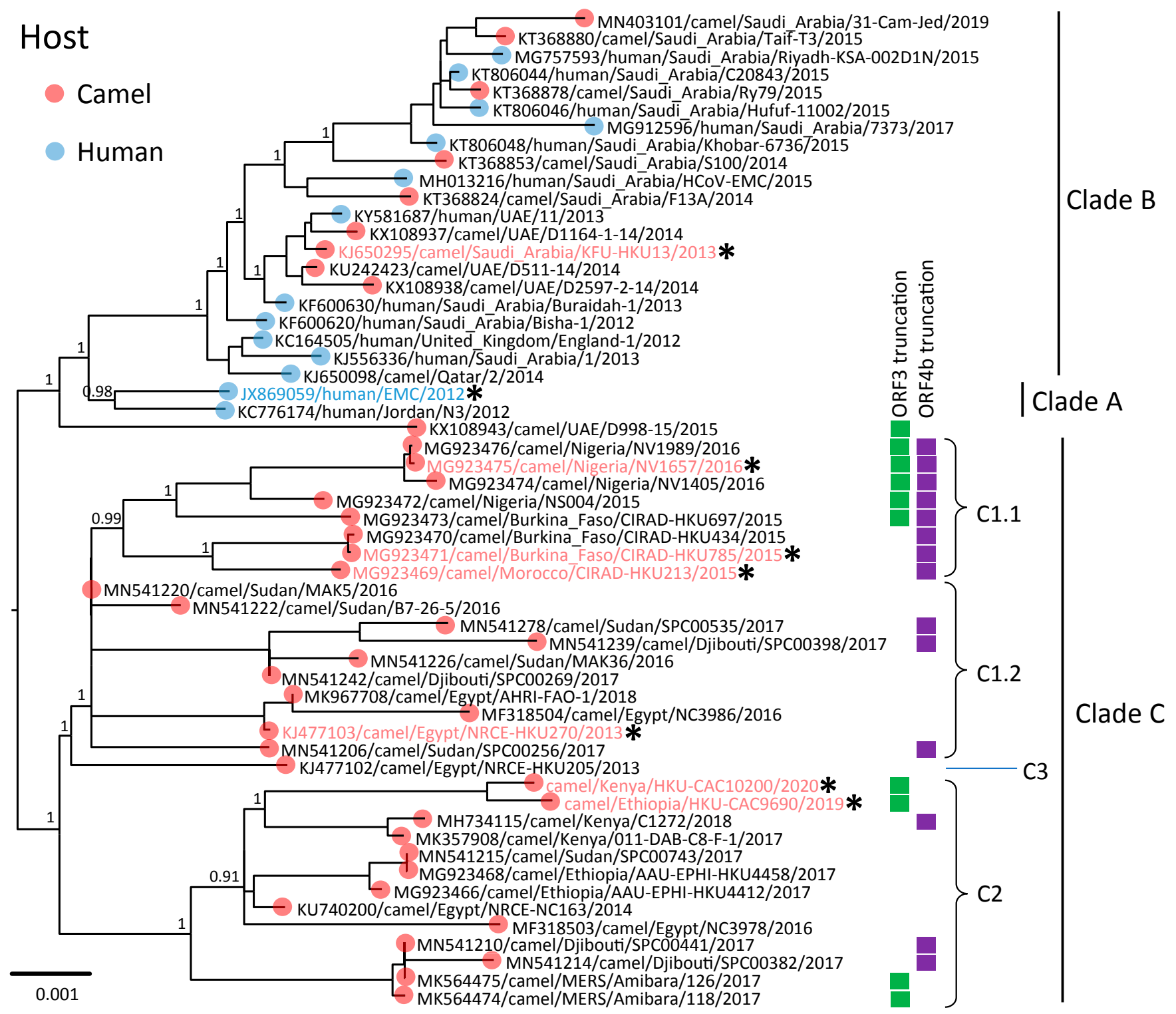

Fig. 1. Phylogenetic relationships of the clade $A, B$, and C MERS-CoVs used in the investigation. Full genome sequences of human MERS-CoV strain (EMC) and camel MERS-CoV strains (AH13, C270, Mor213, BF785, and Nig1657) used in the investigation marked with asterisks are shown to illustrate clade designations and phylogenetic relationships. The tree was constructed by the maximum likelihood method using PhyML. Scale bar indicates the pairwise nucleotide substitutions per site. Taxa labeled with blue and red represent MERS-CoV sequences from human and camels, respectively. ORF3 and ORF4b deletions in the virus genomes are indicated as green and purple boxes, respectively, and more details of the individual deletions are shown in SI Appendix, Table S2. The virus clade designations are denoted.

interspecies transmission, cell tropism, and pathogenesis (22-24). Analysis of the spike protein sequences showed several amino acid differences between the clade $\mathrm{C}$ isolates of MERS-CoVs compared with clade A/B (SI Appendix, Table S4). To examine whether these amino acid substitutions on the spike were a determinant of differences in replication competence between clades $\mathrm{C}$ and $\mathrm{A}$, we generated lentiviruses pseudotyped with spike from EMC (clade A), BF785 (clade C1.1), or Nig1657 (clade C1.1). The two clade $\mathrm{C} 1.1$ viruses had lower virus entry into the human respiratory epithelial cell line Calu-3 (Fig. $4 A$ )

Next, we used reverse genetics to generate a recombinant EMC MERS-CoV (clade A) that carries the spike of EMC or BF785 (clade C1.1). We compared replication kinetics of a reverse genetically derived EMC/2012 virus (rgEMC/2012) with a reverse genetically derived $\mathrm{EMC} / 2012$ with the spike protein of BF785 (rgBF785-S) in Calu-3 cells. The rgBF785-S replicated significantly lower at 24 and $48 \mathrm{~h}$ postinfection compared with the isogenic rgEMC/2012 virus with the EMC spike (rgEMC/ 2012) in Calu-3 cells (Fig. 4B) and ex vivo cultures of human bronchus (SI Appendix, Fig. S4). Human DPP4 knockin mice were infected intranasally with comparable doses of the two viruses $\mathrm{rgBF} 785-\mathrm{S}$ and $\mathrm{rgEMC} / 2012$. At 1 and $3 \mathrm{~d}$ postinfection, viral titers in the lungs were 10 -fold lower in mice infected by the rgBF785-S compared with those infected by the $\mathrm{rgEMC} / 2012$ (Fig. 4C).

Because the spike pseudotype virus experiments suggested that virus spike of clade C1.1 contributed to reduced virus entry, we compared virus entry and infection rates of cells with the recombinant rgBF785-S virus and the isogenic control rgEMC/ 2012 in Calu-3 cells. Calu-3 cells were infected by the recombinant viruses at a multiplicity of infection (MOI) of 5 and stained by monoclonal antibody against the nucleoprotein at $16 \mathrm{~h}$ postinfection 

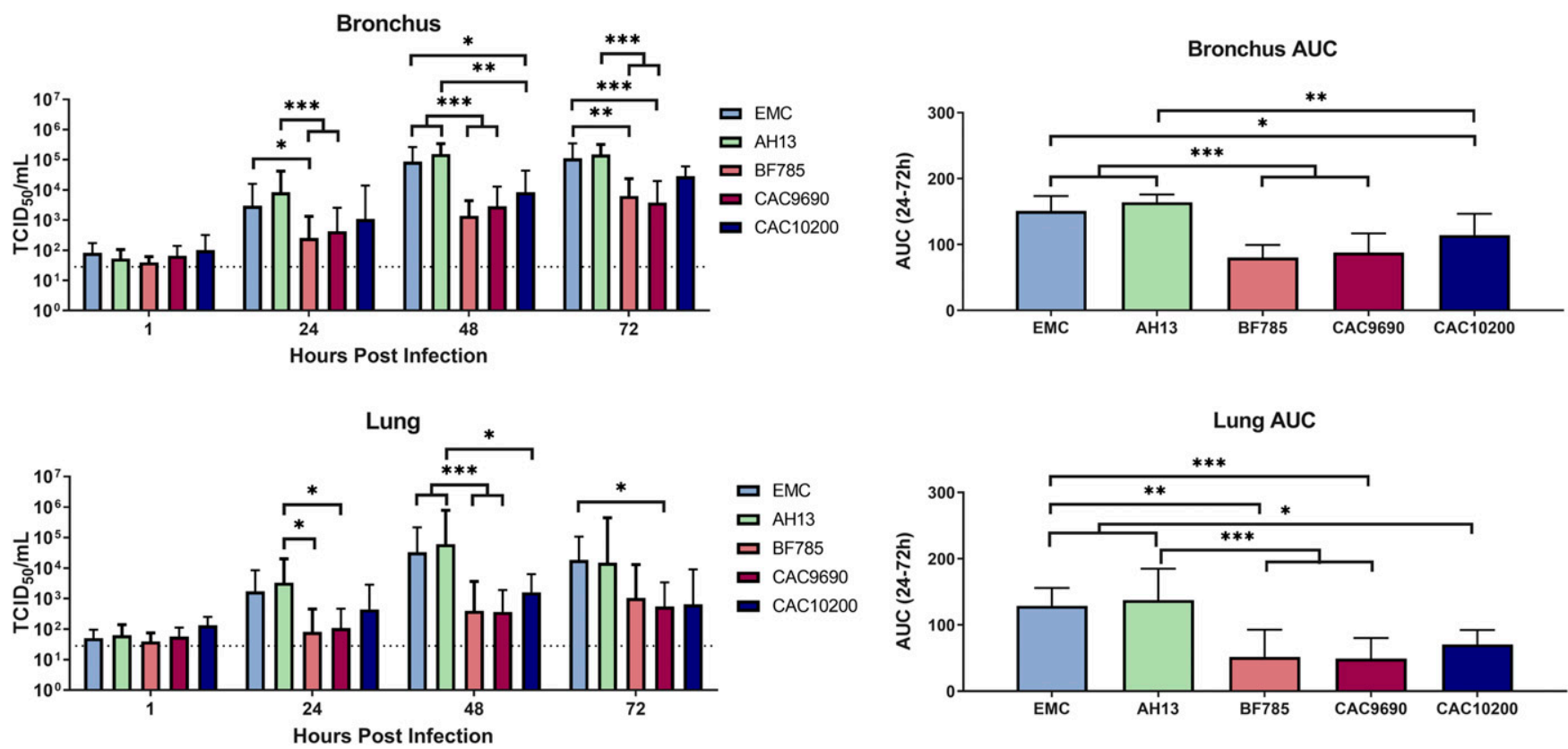

Fig. 2. Comparison of virus replication kinetics in ex vivo cultures of human bronchial and lung tissues. Viral replication kinetics of African viruses BF785 (clade C1.1), CAC9690 (clade C2), and CAC10200 (clade C2) are compared with EMC (clade A) and AH13 (clade B). The bar chart shows the virus titers (TCID 50 ) of culture supernatants at $1,24,48$, and $72 \mathrm{~h}$ postinfection in ex vivo cultures of human bronchus and lung tissues infected with each virus with $10^{6}$ TCID 50 . Data are shown as mean and SD of viral titers from three independent tissue donors. The horizontal dotted line denotes the limit of detection of virus TCID $_{50}$ assay. Statistical significance was determined by two-way ANOVA. Analysis of the 24- to 72-h data are shown on the AUC chart for the bronchus and lung tissues of each virus, and statistical analysis was done using one-way ANOVA. Bonferroni's comparisons were performed. Statistical significance is indicated as follows: ${ }^{*} P<0.05 ; * * P<0.01 ; * * * P<0.001$.

to quantitate the number of cells infected in the first cycle of virus replication. Compared with the rgEMC/2012, the number of cells infected by rgBF785-S was reduced by $49.2 \%$ (Fig. $4 D$ ) (SI Appendix, Fig. S5). We found that there was no visible immunostaining at $8 \mathrm{~h}$ after infection, confirming that we were detecting the first cycle of virus infection at $16 \mathrm{~h}$ postinfection.

The fusogenicity of spike protein was then examined as previously described by transfecting plasmids encoding spike gene of EMC or BF785 in Huh-7 cells (25). The capacity of cell-cell fusion induced by these two $\mathrm{S}$ proteins was similar, with similar size of syncytia (Fig. $4 E$ and SI Appendix, Fig. S6). Taken together, these results indicate that the differences in the spike protein of EMC and BF785 do not lie in their fusogenic properties, but is determined at the initial step of virus attachment and entry.

\section{Discussion}

We had previously demonstrated that clade C.1.1 MERS-CoV from West Africa had reduced virus replication competence in human respiratory cells and ex vivo cultures of human lung compared with clade A or B viruses from Saudi Arabia (18). However, in order to explain the apparent lack of zoonotic MERS disease in Africa, it was essential that this work be extended to geographically and phylogenetically diverse Clade $\mathrm{C}$ viruses from other parts of Africa, especially East Africa which has the highest density of dromedary camels in the world. We now demonstrate that clade $\mathrm{C} 1.1, \mathrm{C} 1.2$, and clade $\mathrm{C} 2$ viruses all have lower replication competence in ex vivo cultures of the human lung and in lungs of hDPP4 knockin mice in comparison with clade A and B viruses found in the Arabian Peninsula.

There is serological evidence that humans are getting infected with MERS-CoV in Africa although seroprevalence appears to be lower than that observed in the Arabian Peninsula (15). It is notable that our recent work on $\mathrm{T}$ cell responses to MERS-CoV in Nigeria (West Africa) demonstrated much higher prevalence $(30 \%)$ of infection in camel-exposed populations than demonstrated by antibody prevalence (26). It is known that humans with asymptomatic or mild MERS-CoV infections fail to make detectable antibody responses $(27,28)$. Thus, MERS-CoV seroprevalence may greatly underestimate the extent of mild or asymptomatic infection taking place in Africa. Taken together with the data from the present study showing lower viral replication competence in the lung, it is plausible that African viruses are infecting humans and eliciting $\mathrm{T}$ cell responses but are unable to cause clinical disease because of the lower replication competence in the lung. These African viruses may be less able to elicit neutralizing antibody because of the asymptomatic or mildly symptomatic nature of the infection. If repeated unsuspected zoonotic transmission of MERS$\mathrm{CoV}$ continues to take place in Africa, given the much larger number of MERS-CoV-infected dromedaries in Africa, the possibility of the virus adapting to efficient transmission between humans is probably more likely in Africa than in the Arabian Peninsula where MERS control efforts have been focused hitherto.

Next, it was important to investigate the viral genetic determinants that influence the difference in phenotype between clade $C$ viruses in Africa and clade $A$ or $B$ viruses associated with pathogenicity in humans in the Arabian Peninsula. Our present investigation showed that the spike gene is one viral determinant leading to the lower replication competence of African isolates in human respiratory epithelial cells. We used lentiviruses pseudotyped with spike of clade A (Saudi Arabia) or clade C1.1 (West Africa) viruses to demonstrate that the clade C1.1 spike is associated with reduced virus entry into Calu-3 cells. We next used reverse genetics to create an EMC virus carrying the spike of BF785 (rgBF785-S) as well as the isogenic virus carrying the EMC spike (rgEMC-S). The rgEMC-S virus had significantly higher replication competence in in vitro, ex vivo human, and in vivo mouse experimental models. Our phenotypic characterization of the rgBF785-S and rgEMC-S viruses suggests that the differences between these viruses are determined at the level of virus entry but not at the stage of virus fusion. 

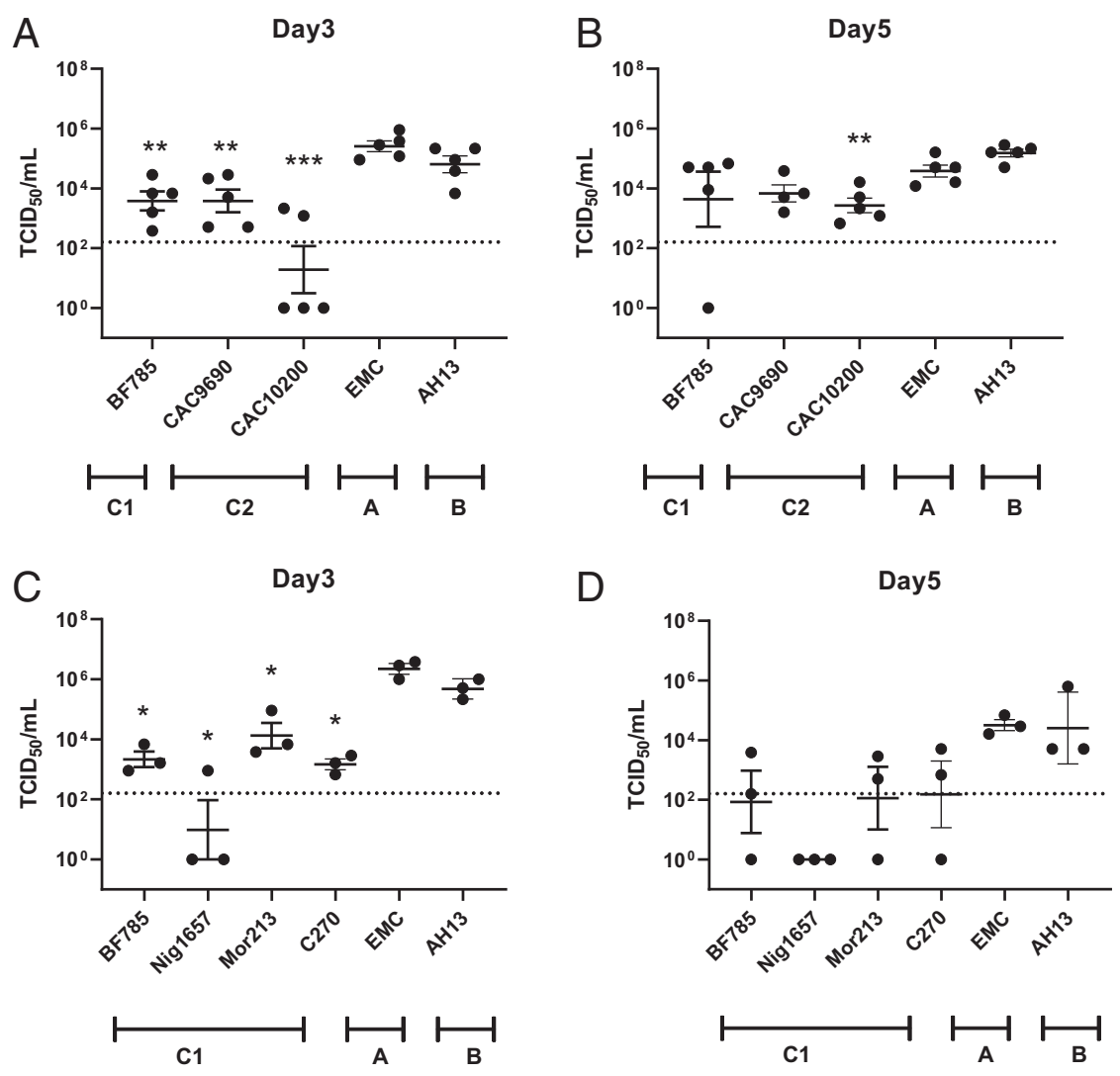

Fig. 3. Comparison of virus replication kinetics in the lung of hDPP4 knockin mice infected by different MERS-CoVs from Saudi Arabia, Burkina Faso, Ethiopia, Kenya, Nigeria, Morocco, and Egypt. Five $(A$ and $B)$ or three $(C$ and $D)$ mice per group ( 6 to 10 wk old, female) were infected intranasally with $10^{4}$ PFU of the respective MERS-CoV strains. Virus titers in the lungs were measured at day $3(A$ and $C)$ and $5(B$ and $D)$ postinfection. Titers are expressed as TCID 50 per milliliter of tissue homogenate. MERS-CoV titers in the lung of mice infected with viruses from Burkina Faso BF785 (clade C1.1), Kenya CAC10200 (clade C2), Ethiopia CAC9600 (clade C2), or Saudi Arabia EMC (clade A) and AH13 (clade B) were compared at day 3 (A) or day 5 (B) postinfection. MERS-CoV strains from Burkina Faso BF785, Nigeria Nig1657 (clade C1.1), Egypt C270 (clade C1.2), and two viruses from Saudi Arabia EMC (clade A) and AH13 (clade B) were compared at day $3(C)$ or $5(D)$ postinfection. Means and SE of means are shown. Statistical significance was determined by comparing the results of EMC with each of the other viruses using Student's $t$ test. ${ }^{*} P<0.05 ; * * P<0.01 ; * * P<0.001$.

The spike protein of African virus BF785 and Nig1657 differ from prototype EMC virus in amino acid residues in the receptor binding domain, but none of them in the receptor binding interface (SI Appendix, Table S4). Amino acid differences V26A and H194Y common to both BF785 and Nig1657 were found at the spike $\mathrm{N}$-terminal domain, which is responsible for binding to sialic acid and is believed to facilitate virus attachment and entry. Amino acid substitution S856Y in the S2 subunit was common to both BF785 and Nig1675, which may potentially affect the fusion efficiency. The role of individual amino acid substitutions or combinations on the observed phenotype deserves further study.

It is important to note that amino acid substitutions in other parts of the genome may also contribute to the phenotypic differences observed. Alignment of the genomes of the viruses studied identifies a number of amino acid substitutions that are unique to the six African viruses studied. With reference to the EMC virus genome, these correspond to ORF1ab amino acid positions N581Y (nsp2), L654F (nsp2), K1066E (nsp3), L1585F (nsp3), I1911T (nsp3), S2000I (nsp3), A2333V (nsp3), F2481C (nsp3), I2639L (nsp3), I2676T (nsp3), and T5546M (nsp13) (Dataset S1). Additionally, Q12H in orf5 also distinguishes the African viruses from the Saudi Arabian viruses EMC and AH13. Factors contributing to the low replication phenotype of African MERS-CoV is likely to be multifactorial, and further studies are needed to completely map the viral genetic determinants of this low replication phenotype.
It is known that naturally occurring deletions in ORF4b, which were found in BF875 virus, may also contribute to a modest reduction in virus replication competence (18). However, such ORF4b deletions were not observed in viruses from East Africa, which also appear to have reduced viral replication competence in human and mouse lung, suggesting that ORF4b deletion is unlikely to be the only determinant of this phenotype.

While our findings suggest that clade $C$ viruses in Africa have lower replication competence in the human lung, possibly explaining the absence of zoonotic MERS in Africa, it is important that more intensive investigations in humans with exposure to dromedary camels in Africa are carried out to look for evidence of human infection and disease. Our previous studies in Nigeria suggest that serological responses are less sensitive than the T cell responses as an indicator for detecting the extent of MERS-CoV infection in humans (26). Such studies need to be confirmed and extended. Studies need to be carried out of people with influenzalike illness (ILI) and severe acute respiratory infections (SARIs) in areas of Africa where interactions between dromedaries and humans are common to confirm the contention that clinically overt MERS is indeed rare. Since the current perception is that MERS disease does not occur in Africa, MERS-CoV is not usually tested for in patients with ILI or SARIs.

It is noted that these clinical outcomes may have multifactorial determinants. While we have shown that a lack of exposure is unlikely to be a major determinant of this apparent difference in clinical outcome (14), it is possible that hitherto unexplored 


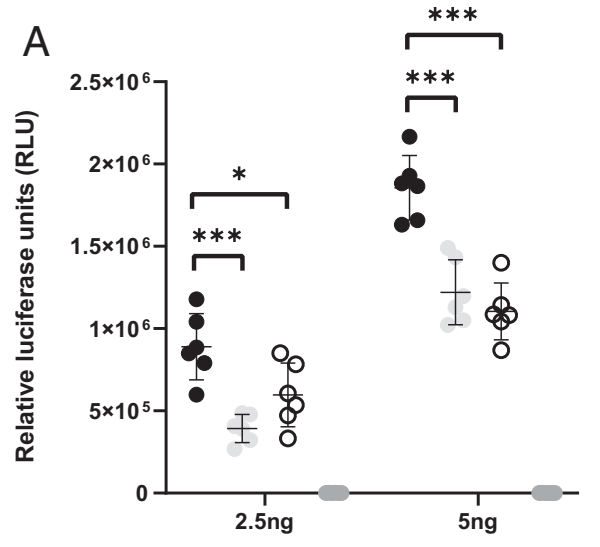

- $\mathrm{EMC/2012pp}$ BF785pp

O Nig1657pp

- Empty vector
B

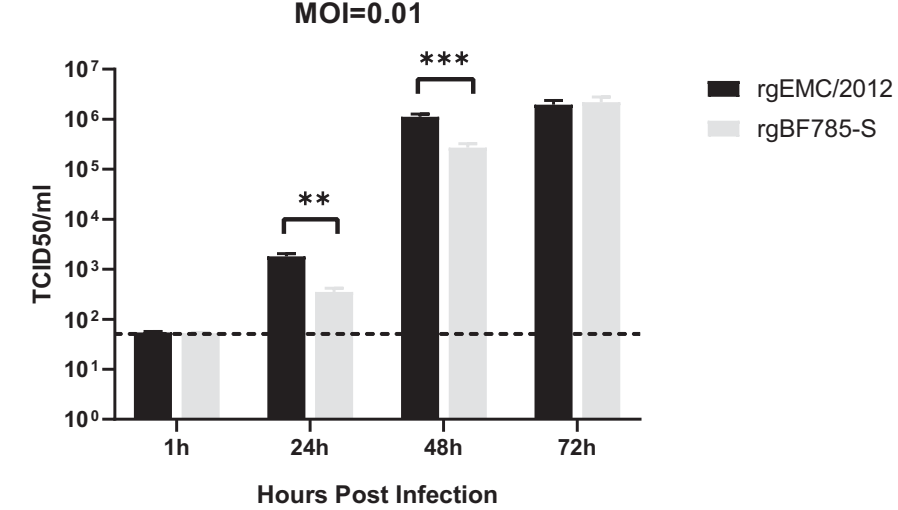

C
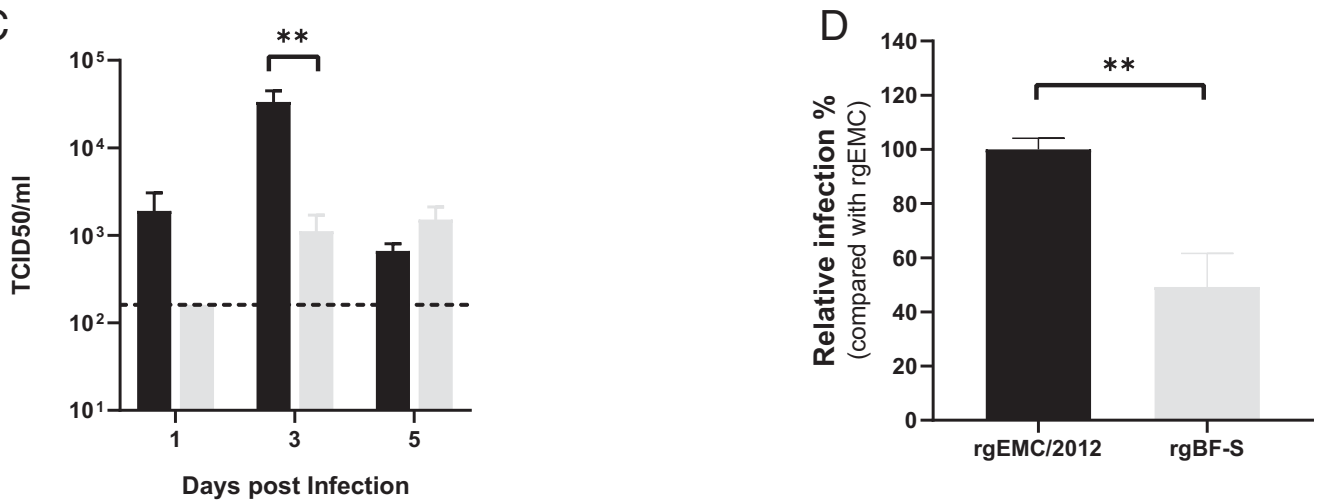

$E$

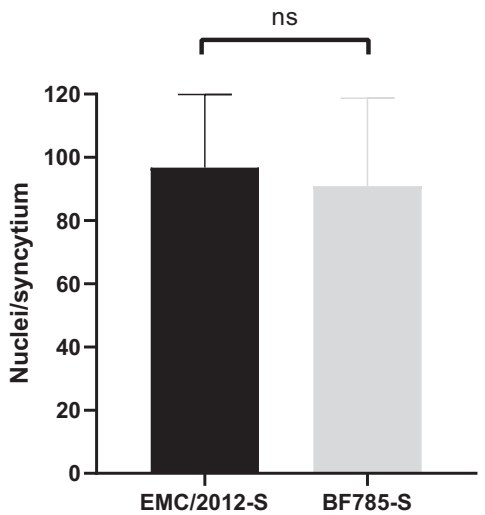

Fig. 4. MERS-CoV spike gene contributes to lower replication competence in clade C MERS-CoVs. (A) Effect of S protein substitutions of BF785 and Nig1657 on cell entry in Calu-3 cells. Calu-3 cells were infected with 2.5 and 5 ng p24 of pseudoparticles carrying S of EMC/2012, BF785, Nig1657, or empty vector. At $66 \mathrm{~h}$ postinfection, cells were lysed and cell entry was measured by luciferase activity. Means and SD of representative experiments were presented. Each experiment was repeated six times. (B) Calu-3 cells were infected at a MOI of 0.01 of rgEMC/2012 and rgBF785-S. Culture supernatants were collected at 1, 24 , 48 , and $72 \mathrm{~h}$ postinfection. Virus titers of the supernatants were determined by the $\mathrm{TCID}_{50}$ assay. Means and SEM of three independent experiments $(n=3)$ are shown. (C) Human DPP4 knockin mice were infected intranasally with $10^{4}$ PFU of the recombinant viruses. Virus titers in the lungs of mice at 1,3 , and $5 \mathrm{~d}$ postinfection were measured by the TCID 50 assay. The virus titers at each time point represent the mean (SE) from four mice. (D) Calu-3 cells were infected with the rgEMC/2012 and rgBF785-S at a MOI of 5 for $16 \mathrm{~h}$. At $16 \mathrm{~h}$ postinfection, the cells were fixed and immunolabeled for nucleoprotein in green and stained for nuclei (DAPI) in blue. Percentages of infected cells were counted. Results are shown as percentages of relative infection (means \pm SD) compared with rgEMC/2012 from three independent experiments $(n=3)$. (E) Huh-7 cells were transfected with plasmids to express S proteins of MERS-CoV BF785 or EMC/2012. After $20 \mathrm{~h}$ postinfection, the cells were fixed and immunolabeled for S proteins in green, and nuclei were stained with DAPI (blue). Nine syncytia were randomly selected for examination from three independent experiments $(n=3)$ and syncytial sizes quantified using Metamorph software. The size of the syncytia are shown as average values of the nuclei/syncytium ratio. Error bars indicate SD. Statistical significance was determined by Student's $t$ test and virus titers were $\log 10$ transformed in analysis. $* * P<0.01 ; * * * P<0.001$. 
human genetic differences may also play an independent or complementing role in viral phenotypic differences.

In summary, our study demonstrates that a genetically diverse range of clade C MERS-CoV from Africa has lower viral replication competence in human and mouse lungs and that this phenotype is contributed to by the virus spike protein, at least in clade $\mathrm{C} 1.1$ virus strains. Given that MERS-CoV is most genetically diverse in Africa and the known trade movements of dromedaries from the Horn of Africa to the Arabian Peninsula (20), it is likely that the pathogenic clade A and B MERS-CoV in the Arabian Peninsula emerged from MERS-CoV in Africa, possibly in recent decades. It has been shown that clade B MERS-CoV now established in the Arabian Peninsula appears to have a fitness advantage in dromedary populations in the Arabian Peninsula, because repeated introduction of clade $\mathrm{C}$ viruses to Saudi Arabia have not resulted in the establishment of these lineages in the Arabian Peninsula (19). It is possible that some of these genetic changes that produced increased fitness for dromedaries fortuitously facilitated increased replication competence in the human lung and pathogenicity in humans. If these pathogenic clade B viruses were to be introduced into dromedary camels in Africa, they may well become dominant in dromedaries in that continent, with major adverse consequences for human health. Since the trade movements are almost uniformly into the Arabian Peninsula rather than out of it $(19,20)$, this risk may not be high at present, but it is important to be aware of it and mitigate such an eventuality. Alternatively, MERS-CoVs in Africa may independently acquire amino acid substitutions in the spike that may confer increased pathogenicity for humans. Either scenario would lead to major adverse health impacts in Africa and be associated with pandemic threat. The findings thus highlight the need for enhanced MERS$\mathrm{CoV}$ surveillance in dromedaries and humans in Africa and for measures to avoid introduction of clade B viruses from the Arabian Peninsula to Africa.

\section{Materials and Methods}

Virus. Viruses used in this study, virus abbreviations, host and country of origin, sampling date, clade designation, and GenBank accession numbers are listed in SI Appendix, Table S1. MERS-CoV human/EMC/2012 (clade A) (EMC) was the prototype MERS-CoV, kindly provided by Ron Fouchier, Erasmus MC Rotterdam, The Netherlands (7). The specimen collection details and genetic characterization of the camel viruses from Burkina Faso (BF785) (clade C1.1) Nigeria (Nig1657) (clade C1.1), Morocco (MOR213) (clade C1.1), Egypt (C270) (clade $\mathrm{C1.2}$ ), and Saudi Arabia (AH13) have been previously reported (18, 29). The viruses from Kenya, camel/Kenya/CAC10200/2020 (clade C2) and Ethiopia, camel/Ethiopia/CAC9690/2020 (clade C2) were detected and isolated as part of this study. All the dromedary camel viruses used in this study were isolated in our laboratory in Vero cells (ATCC CCL-81) and were passaged, aliquoted, and stored at $-80^{\circ} \mathrm{C}$ until used.

Ex Vivo Cultures of Human Bronchus and Lung Tissues. The method for preparing ex vivo human bronchus and lung tissue was reported in detail in our previous study (29) using F12K culture medium (Thermo Fisher) with 100 units $/ \mathrm{mL}$ penicillin and $1 \mathrm{mg} / \mathrm{mL}$ streptomycin (Life Technologies). Informed consent was obtained from patients undergoing surgical resection of lung tissue at Queen Mary Hospital as part of routine clinical care for the use of fragments of fresh human bronchus and lung, surplus to diagnostic requirements, for the purpose of research. This study was approved by the Institutional Review Board of The University of Hong Kong/Hospital Authority Hong Kong West Cluster (UW-13-104 and UW-20-862). Tissues from each donor were tested in duplicate and data from three donors were used as biological replicates. Bronchial and lung tissues cultured ex vivo were infected with MERS-CoVs by incubating $1 \mathrm{~mL}$ of each virus at a titer of $10^{6}$ $\mathrm{TCID}_{50} / \mathrm{mL}$ at $37^{\circ} \mathrm{C}$ for $1 \mathrm{~h}$. The tissues were then washed three times with phosphate budffered saline (PBS) (Thermo Fisher), replenished with the cell culture medium Dulbecco's minimum essential medium (DMEM) (Thermo Fisher), and incubated for $72 \mathrm{~h}$ in $5 \% \mathrm{CO}_{2}$ at $37{ }^{\circ} \mathrm{C}$. Uninfected tissue fragments incubated with DMEM served as negative controls. Culture super natants from the infected tissues were collected at 1, 24, 48, and $72 \mathrm{~h}$ postinfection and titrated in parallel for infectious virus using TCID $_{50}$ assays in Vero cells. The virus titers at 24,48 , and $72 \mathrm{~h}$ were used to derive an area under the curve (AUC) as a composite measure of the replication competence of each virus (30). At $72 \mathrm{~h}$ postinfection, ex vivo tissue fragments were fixed in $10 \%$ formalin and processed for immunohistochemistry to detect MERS-CoV antigen expression (see ref. 29 for detailed methods).

Experimental Infection of hDPP4 Knockin Mice. The hDPP4 knockin mouse model was generated by humanizing exons 10 to 12 of the mouse DPP4 locus (the region of the hDPP4 molecule that is crucial for MERS-CoV receptor binding) (21). Specific pathogen-free hDPP4 knockin mice in a B6 background were obtained from Stanley Perlman, Department of Microbiology and Immunology, University of lowa, lowa City, IA and maintained in the Laboratory Animal Unit at The University of Hong Kong. The experiments were conducted in the biosafety level 3 (BSL3) facility at The University of Hong Kong. The study protocol was carried out in strict accordance with recommendations and approved by the Committee on the Use of Live Animals in Teaching and Research of The University of Hong Kong (CULATR 4534-17). The hDPP4 mice aged 6 to $10 \mathrm{wk}$ were lightly anesthetized with ketamine and infected intranasally with $10^{4}$ plaque-forming units (PFU) of each MERS-CoV subtype in $25 \mu \mathrm{L}$ PBS. Three mice from each group were killed at 1,3 , and $5 \mathrm{~d}$ postinfection, respectively. Virus titers in the lungs were determined by the $\operatorname{TCID}_{50}$ assays in Vero cells.

Generation of MERS-CoV Spike Virus Pseuotyped Lentiviruses and Pseudotype Assays for Virus Entry. We used the method described by us previously to generate lentivirus pseudotypes expressing a mammalian codon-optimized EMC/2012 spike (GenBank: AFS88936.1) pcDNA3.1(+) (31). Multi site-directed mutagenesis (Agilent) was used to introduce the substitutions to mimic the $S$ protein of BF785 (GenBank: AVN89344.1) (V26A, A89S, H194Y, T424I, S856Y, and A1158S) and Nig1657 (GenBank: AVN89387.1) (V26A, H167Y, H194Y, L495F, L588F, S856Y, A1158L, and L1200F). Sanger sequencing was performed to verify the mutated sites.

HIV/MERS spike pseudoparticles containing S protein of EMC/2012, BF785, and Nig1657 were generated as reported previously (31). pNL Luc E- R-vector encoding the luciferase reporter gene and pcDNA-S plasmids (EMC/2012, BF785, and Nig1657) or an empty vector were cotransfected in 293T cells using TransIT-LT1 transfection reagent (Mirus). After $48 \mathrm{~h}$, supernatants were harvested and concentrated and pseudoparticles were quantified using HIV p24 viral protein by p24 antigen ELISA kit (ZeptoMetrix) to quantitate the HIV core of the psudotyped viruses. Pseudoparticles of $50 \mu \mathrm{L}$ with $2.5 \mathrm{ng}$ and $5 \mathrm{ng} \mathrm{p} 24$ were used to infect Calu-3 cells $\left(1 \times 10^{4}\right.$ cells/well in 96-well cell plates) and incubated for $18 \mathrm{~h}$. One hundred microliters of complete cell growth medium was added and cells were incubated for an additional $48 \mathrm{~h}$. Cells were then lysed by adding lysis buffer and luciferase substrate (Promega). Luciferase activity was measured using the Microbeta Luminometer (PerkinElmer).

Generation of Recombinant Wild-Type and Mutant MERS-CoVs. A MERS-CoV infectious bacterial artificial chromosome (BAC) clone of EMC/2012 was used as a template. To generate a chimeric plasmid encoding the sequence of $\mathrm{EMC} / 2012$ carrying the full spike gene of BF785, the Gibson assembly technique was used (32). A linearized vector was first generated by removing the spike gene in the EMC/2012 plasmid using restriction enzymes, Swal and Pacl (NEB). The BF785 spike gene was generated by chemical synthesis (Sangon Biotech) and amplified by PCR with primers containing overlapping sequence of the linearized vector (MERS-BF-spike-forward: AAAACGACGGCCAGTATTTAAATGAGTTCG, MERS-BF-spike-reverse: GACCATGATTACGCCTTAATTAACTGAGTA). The PCR product was then cloned into the linearized vector using a Gibson assembly cloning kit (NEB) to generate the mutant construct. EMC/2012 and its mutant constructs were transfected in BHK21 (ATCC CCL-10) cells individually using Lipofectamine 2000 (Thermo Fisher). At $6 \mathrm{~h}$ posttransfection, cells were trypsinized and reseeded in Huh-7 cells. Supernatants were collected at $48 \mathrm{~h}$ posttransfection and the viruses were further propagated only once in Huh-7 cells. The recombinant EMC/2012 and the mutant were verified, followed by plaque purification and genetic characterization using next generation sequencing (as detailed in ref. 18).

Virus Replication in Calu-3 Cells. Experiments for the comparison of viral replication kinetics in Calu-3 cells (ATCC HTB55) were performed as previously described (18). A total of $1.5 \times 10^{5}$ Calu-3 cells per well were seeded in 24-well tissue culture plates (TPP Techno Plastic Products) and infected with $\mathrm{rgEMC} / 2012$ and $\mathrm{rgBF} 785-\mathrm{S}$ with $\mathrm{MOI}=0.01$. Aliquots of supernatant was collected at $1,24,48$, and $72 \mathrm{~h}$ postinfection and titrated by $\mathrm{TCID}_{50}$ using Vero cells. For immunofluorescence staining, the Calu-3 cells were infected with the recombinant MERS-CoVs at MOI of 5 . The cells were fixed with $4 \%$ paraformaldehyde (PFA) (Biotium) at $16 \mathrm{~h}$ postinfection, permeabilized with 
$0.3 \%$ Triton X-100 (Sigma-Aldrich), and immunolabeled using primary polyclonal antibody against MERS-CoV nucleoprotein (Abbiotec) and secondary Alexa Fluor 488-conjugated goat anti-rabbit antibody (Thermo Fisher). Nuclei were stained by DAPI (Thermo Fisher). Images were acquired by a Zeiss LSM710 inverted confocal microscope (Carl Zeiss) with a 10x objective. The percentage of infected cells was analyzed using the cell scoring function on the Metamorph software (Molecular Devices).

Cell-Cell Fusion Assay. A total of $1.25 \times 10^{5}$ Huh-7 cells were first seeded on coverslips in 24-well plates (TPP Techno Plastic Products) for $24 \mathrm{~h}$. Plasmids encoded with the spike of EMC/2012, BF785, or an empty vector were transfected in the cells using TransIT-LT1 transfection reagent (Mirus) (25). At $20 \mathrm{~h}$ posttransfection, the cells were fixed with $4 \%$ PFA, permeabilized with $0.3 \%$ Triton $\mathrm{X}-100$, and immunolabeled using primary polyclonal an tibody against MERS-CoV spike protein (SinoBiological) and secondary Alexa Fluor 488-conjugated goat anti-rabbit antibody (Thermo Fisher). Nuclei were stained using DAPI. Images were acquired by a Zeiss LSM710 inverted confocal microscope with a 20x objective. Cell scoring function in Metamorph software was used to quantify syncytia formation by calculating nuclei/ syncytium.

Phylogenetic Analysis. Representative and complete MERS-CoV genome sequences from predesignated clades $(A, B$, and $C)$ were downloaded from GenBank and aligned by MAFFT. The phylogenetic tree was generated using the maximum likelihood method (PhyML) (18).

Statistical Analysis. Student's $t$ test and one-way and two-way ANOVA with Bonferroni's comparison were used to compare difference of mean values

1. P. Zhou et al., A pneumonia outbreak associated with a new coronavirus of probable bat origin. Nature 579, 270-273 (2020).

2. T. T. Lam et al., Identifying SARS-CoV-2-related coronaviruses in Malayan pangolins. Nature 583, 282-285 (2020).

3. L. Vijgen et al., Complete genomic sequence of human coronavirus OC43: Molecular clock analysis suggests a relatively recent zoonotic coronavirus transmission event J. Virol. 79, 1595-1604 (2005).

4. V. M. Corman et al., Link of a ubiquitous human coronavirus to dromedary camels Proc. Natl. Acad. Sci. U.S.A. 113, 9864-9869 (2016)

5. J. S. M. Peiris, Y. Guan, K. Y. Yuen, Severe acute respiratory syndrome. Nat. Med. 10(suppl. 12), S88-S97 (2004)

6. S. K. Lau et al., Severe acute respiratory syndrome coronavirus-like virus in Chinese horseshoe bats. Proc. Natl. Acad. Sci. U.S.A. 102, 14040-14045 (2005)

7. A. M. Zaki, S. van Boheemen, T. M. Bestebroer, A. D. Osterhaus, R. A. Fouchier, Isolation of a novel coronavirus from a man with pneumonia in Saudi Arabia. N. Engl. J. Med. 367, 1814-1820 (2012).

8. E. I. Azhar et al., Evidence for camel-to-human transmission of MERS coronavirus. N. Engl. J. Med. 370, 2499-2505 (2014).

9. Korea Centers for Disease Control and Prevention, Middle East respiratory syndrome coronavirus outbreak in the Republic of Korea, 2015. Osong Public Health Res. Perspect. 6, 269-278 (2015)

10. H. H. Balkhy et al., Notes from the field: Nosocomial outbreak of Middle East respiratory syndrome in a large tertiary care hospital-Riyadh, Saudi Arabia, 2015. MMWR Morb. Mortal. Wkly. Rep. 65, 163-164 (2016)

11. M. S. Mehand, F. Al-Shorbaji, P. Millett, B. Murgue, The WHO R\&D blueprint: 2018 review of emerging infectious diseases requiring urgent research and development efforts. Antiviral Res. 159, 63-67 (2018).

12. E. Miguel et al., Risk factors for MERS coronavirus infection in dromedary camels in Burkina Faso, Ethiopia, and Morocco, 2015. Euro Surveill. 22, 30498 (2017).

13. R. S. Sikkema et al., Global status of Middle East respiratory syndrome coronavirus in dromedary camels: A systematic review. Epidemiol. Infect. 147, e84 (2019).

14. R. T. So et al., Lack of serological evidence of Middle East respiratory syndrome coronavirus infection in virus exposed camel abattoir workers in Nigeria, 2016. Euro Surveill. 23, 1800175 (2018).

15. A. N. Alshukairi et al., High prevalence of MERS-CoV infection in camel workers in Saudi Arabia. mBio 9, e01985-e18 (2018).

16. A. Abbad et al., Middle East respiratory syndrome coronavirus (MERS-CoV) neutralising antibodies in a high-risk human population, Morocco, November 2017 to January 2018. Euro Surveill. 24, 1900244 (2019). among groups of continuous variables. Statistical analyses were done with GraphPad Prism version 8.

Data Availability. All study data are included in the article and/or supporting information.

ACKNOWLEDGMENTS. We acknowledge Dr. Jean Millet for advice on the MERS-CoV spike fusion assays and Dr. Eric H. Y. Lau for advice on statistica analysis. We acknowledge the technical assistance of Dr. B. Getachew, Mr. K. Adamu, Mrs. H. Mohammed (National Veterinary Institute, Ethiopia) Drs. O. Njagi, G. Njogu, L. Wangui, J. Ngoci, and W. Mbaya (Central Veterinary Laboratory, Kenya), Dr. T. Nyariki (National MERS-CoV Coordinator, Food and Agriculture Organization [FAO], Kenya), and Drs. Gijs van 't Klooster, Nega Tewolde, and G. Gari (FAO Emergency Centre for Transboundary Animal Diseases [ECTAD] Program, Ethiopia). We acknowledge the support of Yilma Makonnen, Emma Garder, Ryan Aguanno, Cristian De Battisti, and Agneleque Angot (FAO of the United Nations). We thank M. C. Cheung, Rachel H. H. Ching, John C. W. Ho, Rita Lai, L. K. Chi, and Keith Chan (The University of Hong Kong) for technical assistance. The research was funded by the Commissioned Research on Control of Infectious Diseases (phase III and IV) from the Health and Medical Research Fund, Hong Kong Special Administrative Region; Health and Medical Research Fund Grant 19181032; NIH (Contract HHSN272201400006C and U01 Grant Al151810), a Calmette and Yersin scholarship from the Pasteur International Network Association, and Guangdong Province International Scientific and Technological Cooperation Projects (Grant 2020A0505100063). Field studies in Kenya and Ethiopia were supported through the FAO of the United Nations project OSRO/GLO/505/USA, which is funded by the US Agency for International Development (USAID). The views and opinions expressed in this paper are those of the authors and are not necessarily the views of USAID or FAO.

17. A. Liljander et al., MERS-CoV antibodies in humans, Africa, 2013-2014. Emerg. Infect Dis. 22, 1086-1089 (2016)

18. D. K. W. Chu et al., MERS coronaviruses from camels in Africa exhibit regiondependent genetic diversity. Proc. Natl. Acad. Sci. U.S.A. 115, 3144-3149 (2018)

19. S. A. El-Kafrawy et al., Enzootic patterns of Middle East respiratory syndrome coronavirus in imported African and local Arabian dromedary camels: A prospective genomic study. Lancet Planet. Health 3, e521-e528 (2019).

20. V. Alary, B. Faye, The camel chains in East Africa-Importance of gaps between the data and the apparent reality. J. Camelid Sci. 9, 1-22 (2016).

21. K. Li et al., Mouse-adapted MERS coronavirus causes lethal lung disease in human DPP4 knockin mice. Proc. Natl. Acad. Sci. U.S.A. 114, E3119-E3128 (2017)

22. F. Li, Structure, function, and evolution of coronavirus spike proteins. Annu. Rev. Virol. 3, 237-261 (2016)

23. S. Belouzard, J. K. Millet, B. N. Licitra, G. R. Whittaker, Mechanisms of coronavirus cell entry mediated by the viral spike protein. Viruses 4, 1011-1033 (2012).

24. R. L. Graham, R. S. Baric, Recombination, reservoirs, and the modular spike: Mechanisms of coronavirus cross-species transmission. J. Virol. 84, 3134-3146 (2010).

25. J. K. Millet et al., A camel-derived MERS-CoV with a variant spike protein cleavage site and distinct fusion activation properties. Emerg. Microbes Infect. 5, e126 (2016)

26. C. K. P. Mok et al., Specific T cell responses provide evidence of MERS coronavirus infection in people with occupational exposure to dromedary camels in Nigeria. Lancet Infect. Dis. 21, 385-395 (2020)

27. C. Drosten et al., Transmission of MERS-coronavirus in household contacts. N. Engl. J. Med. 371, 828-835 (2014)

28. P. G. Choe et al., MERS-CoV antibody responses 1 year after symptom onset, South Korea, 2015. Emerg. Infect. Dis. 23, 1079-1084 (2017).

29. R. W. Chan et al., Tropism and replication of Middle East respiratory syndrome coronavirus from dromedary camels in the human respiratory tract: An in-vitro and ex vivo study. Lancet Respir. Med. 2, 813-822 (2014).

30. K. P. Hui et al., Tropism and innate host responses of influenza A/H5N6 virus: An analysis of ex vivo and in vitro cultures of the human respiratory tract. Eur. Respir. J. 49, 1601710 (2017)

31. R. A. Perera et al., Seroepidemiology for MERS coronavirus using microneutralisation and pseudoparticle virus neutralisation assays reveal a high prevalence of antibody in dromedary camels in Egypt, June 2013. Euro Surveill. 18, pii=20574 (2013).

32. A. M. Nikiforuk et al., Rapid one-step construction of a Middle East respiratory syndrome (MERS-CoV) infectious clone system by homologous recombination. J. Virol. Methods 236, 178-183 (2016). 